\title{
Video Article \\ The Infiltration-centrifugation Technique for Extraction of Apoplastic Fluid from Plant Leaves Using Phaseolus vulgaris as an Example
}

\author{
Brendan M. O'Leary ${ }^{1}$, Arantza Rico ${ }^{2}$, Sarah McCraw ${ }^{1}$, Helen N. Fones ${ }^{3}$, Gail M. Preston ${ }^{1}$ \\ ${ }^{1}$ Department of Plant Sciences, University of Oxford \\ ${ }^{2}$ School of Education of Vitoria-Gasteiz, University of the Basque Country (UPV/EHU) \\ ${ }^{3}$ Biosciences, University of Exeter
}

Correspondence to: Gail M. Preston at gail.preston@plants.ox.ac.uk

URL: https://www.jove.com/video/52113

DOI: doi: $10.3791 / 52113$

Keywords: Plant Biology, Issue 94, Apoplast, apoplast washing fluid, plant leaves, infiltration-centrifugation, plant metabolism, metabolomics, gas chromatography-mass spectrometry

Date Published: $12 / 19 / 2014$

Citation: O'Leary, B.M., Rico, A., McCraw, S., Fones, H.N., Preston, G.M. The Infiltration-centrifugation Technique for Extraction of Apoplastic Fluid from Plant Leaves Using Phaseolus vulgaris as an Example. J. Vis. Exp. (94), e52113, doi:10.3791/52113 (2014).

\section{Abstract}

The apoplast is a distinct extracellular compartment in plant tissues that lies outside the plasma membrane and includes the cell wall. The apoplastic compartment of plant leaves is the site of several important biological processes, including cell wall formation, cellular nutrient and water uptake and export, plant-endophyte interactions and defence responses to pathogens. The infiltration-centrifugation method is well established as a robust technique for the analysis of the soluble apoplast composition of various plant species. The fluid obtained by this method is commonly known as apoplast washing fluid (AWF). The following protocol describes an optimized vacuum infiltration and centrifugation method for AWF extraction from Phaseolus vulgaris (French bean) cv. Tendergreen leaves. The limitations of this method and the optimization of the protocol for other plant species are discussed. Recovered AWF can be used in a wide range of downstream experiments that seek to characterize the composition of the apoplast and how it varies in response to plant species and genotype, plant development and environmental conditions, or to determine how microorganisms grow in apoplast fluid and respond to changes in its composition.

\section{Video Link}

The video component of this article can be found at https://www.jove.com/video/52113/

\section{Introduction}

The plant apoplast is the intercellular space that surrounds plant cells. This is a dynamic environment in which many metabolic and transport processes take place. The major structural component of the apoplast is the cell wall, which is assembled and modified by enzymes, structural proteins and metabolites located within the apoplast. In healthy plant cells the apoplast is generally maintained in an acidic state allowing amino acids, sugars and other nutrients to be imported from the apoplast to the cytoplasm by $\mathrm{H}^{+}$symport ${ }^{1}$. During sucrose transport, sucrose moves from photosynthetic sources through the apoplast and into phloem vasculature; sucrose is then transported by an osmotic potential maintained by apoplastic invertases cleaving sucrose in the sink organs ${ }^{2}$. Sugars and other nutrients can also be transported upwards and accumulate in stomatal cavities through the transpiration stream ${ }^{3}$.

The apoplast also represents an environmental niche where many pathogens establish their parasitic lifestyle. Bacterial plant pathogens have the ability to multiply to high densities in the apoplast, which they access through natural openings such as stomata or through wounds ${ }^{4}$. The concentration of amino acids and other nitrogen compounds in tomato apoplast have been shown to be sufficient to support the nutritional requirements of bacterial and fungal pathogens ${ }^{5,6}$. Primary defense responses towards microbial pathogens also occur in the apoplast, namely the production of reactive oxygen species by extracellular peroxidases and oxidases and the strengthening of the cell wall through crosslinking and callose deposition ${ }^{\top}$. The plant cell wall is rich in secondary metabolites with anti-microbial activities; the biochemical nature of these secondary metabolites varies between species ${ }^{8}$.

As a result of the above-mentioned and other processes, leaf apoplastic fluid contains a variety of proteins, sugars, organic acids, amino acids, secondary metabolites, metals and other cations (e.g., $\mathrm{Mg}^{2+}, \mathrm{K}^{+}, \mathrm{Na}^{+}, \mathrm{Ca}^{2+}, \mathrm{Fe}^{2 / 3+}$ ). Solute concentrations in the apoplast of shoots are controlled largely by the balance of transport processes occurring between the apoplast and the xylem, phloem and cytoplasm ${ }^{9}$. However, metabolic reactions and microbial growth also consume or produce apoplastic solutes. The composition of the apoplast is known to differ between plant species and genotypes and in response to changing environmental conditions including light, nutrition and biotic and abiotic stresses ${ }^{9}$. By studying the composition of the apoplast and how it changes, including properties such as redox and osmotic potential, $\mathrm{pH}$, nutrient/metabolite availability and enzymatic activities, one can gain novel insights into how plants respond to their environment. Understanding or characterizing the molecular changes that occur in the apoplast solution is complicated because it is a spatially structured and dynamic compartment in which metabolites and/or ions may be volatile, transient or associated with the cell wall and plasma membrane. Furthermore, different analytical techniques are required to cover the range of different chemical types. 
To study the composition of the soluble apoplast, fluid usually needs to be extracted from the tissue. Several methods exist to extract apoplast fluid from various tissues, including a newly proposed filter strip method ${ }^{10}$, but the most established extraction method for leaves is infiltrationcentrifugation. This technique has been evaluated previously ${ }^{11-13}$ and Lohaus et al. $2001^{14}$ provide a thorough examination of many of the method's technical parameters. As implied by the name, the infiltration-centrifugation technique is a two-step method that essentially involves replacement of the apoplastic air space with an aqueous infiltration fluid, which mixes with the native apoplastic fluid, followed by recovery of the infiltration/apoplastic fluid mixture by gentle centrifugation of the leaves. As the recovered fluid is diluted and does not contain all compounds present in the apoplast (see below), this fluid is commonly known as apoplast washing fluid (AWF), or sometimes intercellular washing fluid, rather than apoplastic fluid. The infiltration-centrifugation technique is easily scalable allowing single or pooled AWF samples of adequate volume to be generated and subjected to a wide range of downstream biochemical and analytical techniques (e.g., protein electrophoresis, enzyme activity measurement, NMR, many types of chromatography and mass spectrometry). Leaf AWF is also useful as an apoplast mimicking growth medium for studying the interaction of plant colonizing microbes with their environment ${ }^{5}$.

In the following protocols we describe how to perform the infiltration-centrifugation technique using Phaseolus vulgaris cv. Tendergreen leaves, and provide some examples of downstream analyses with a focus on metabolomics. Importantly, methods to assess the quality of AWF are provided along with advice to optimize the procedure for different leaf types.

\section{Protocol}

NOTE: The choice of starting leaf material and standardization of plant growth conditions is critical as these factors can greatly affect the quality, variability and yield of AWF (Figure 1). Parameters to consider are shown in Table 1 and are discussed in more detail in the discussion.

\begin{tabular}{|c|c|c|c|}
\hline \multirow[t]{2}{*}{ Parameter } & \multicolumn{3}{|l|}{ Example standardizations } \\
\hline & Phaseolus vulgaris & Solanum lycopersicum & Arabidopsis thaliana \\
\hline Leaf type & $\begin{array}{l}\text { First true leaves, fully expanded, } \\
\text { healthy }\end{array}$ & $\begin{array}{l}2^{\text {nd }} \text { and } 3^{\text {rd }} \text { leaves starting from } \\
\text { bottom, } 4 \text { largest leaflets taken } \\
\text { apical leaflet not used for apoplast } \\
\text { extraction }\end{array}$ & Fully expanded rosette leaves \\
\hline Plant age & 21 days & 7 - 9 weeks & 7 weeks \\
\hline Time of day & Middle of light period (12:00) & Middle of light period (12:00) & Middle of light period (12:00) \\
\hline Hydration & $\begin{array}{l}\text { All plants well watered } 1 \mathrm{hr} \text { pre- } \\
\text { harvest }\end{array}$ & $\begin{array}{l}\text { All plants well watered } 1 \mathrm{hr} \text { pre- } \\
\text { harvest }\end{array}$ & $\begin{array}{l}\text { All plants well watered } 1 \mathrm{hr} \text { pre- } \\
\text { harvest }\end{array}$ \\
\hline Light & $16 \mathrm{hr}$ light, $400 \mu \mathrm{mol} \mathrm{m}{ }^{-2} \mathrm{sec}^{-1}$ & $16 \mathrm{hr}$ light, $400 \mu \mathrm{mol} \mathrm{m}{ }^{-2} \mathrm{sec}^{-1}$ & $\begin{array}{l}10 \mathrm{hr} \text { light (short day) from week } 2 \text {, } \\
400 \mu \mathrm{mol} \mathrm{m}^{-2} \mathrm{sec}^{-1}\end{array}$ \\
\hline Humidity & $70 \%$ & $70 \%$ & $70 \%$ \\
\hline Temperature & $22^{\circ} \mathrm{C}$ light, $18^{\circ} \mathrm{C}$ dark & $22^{\circ} \mathrm{C}$ light, $18^{\circ} \mathrm{C}$ dark & $21^{\circ} \mathrm{C}$ \\
\hline
\end{tabular}

Table 1. Examples of standardized parameters for leaves used in AWF extractions.

\section{Generating Apoplast Washing Fluid}

NOTE: During this protocol hazardous materila inculding microbial pathogens from infected leaves should not be washed down the drain; rather proper disposal procedures should be used.

1. Choose apparatus based on leaf size:

1. Infiltrate smaller leaves (e.g., Arabidopsis, bean) using a needleless syringe. Use a vacuum flask to infiltrate leaves that are too large to fit in a syringe. Use the vacuum flask method to infiltrate multiple leaves simultaneously.

2. Divide leaves that are too large for the available infiltration method into smaller sections using a razor blade. Thoroughly rinse cut leaf sections in distilled water prior to infiltration to remove cytoplasmic contaminants that exude from damaged cells.

2. Leaf infiltration using a $60 \mathrm{ml}$ syringe:

1. Detach the leaf from the plant at the petiole using a razor blade. For compound leaves such as tomato, detach leaflets at the petiolule.

2. Rinse the freshly excised leaf by submersion in distilled water to remove leaf surface contaminants. Dry the leaf by gently blotting with absorbent tissue or paper.

3. Measure the leaf weight before infiltration.

4. Carefully roll and/or fold the leaf into a $60 \mathrm{ml}$ syringe and fill the syringe with distilled water (other infiltration fluids may be used, see discussion). Eject any air within the syringe while lowering the plunger to approximately the $40 \mathrm{ml}$ mark.

5. Cover the syringe tip with either a gloved finger or a piece of Parafilm, then create negative pressure within the syringe by pulling the plunger outwards to the $60 \mathrm{ml}$ mark. Slowly release the plunger.

NOTE: Quickly releasing the pressure may result in cell lysis and cytoplasmic contamination.

6. Unplug the syringe tip and eject any air, then replug the tip and press further downwards on the plunger to create a modest amount of positive pressure.

7. Repeat steps 1.2.5 - 1.2.6 until the leaf is fully infiltrated, as seen by the darkened colour of the infiltrated areas.

8. Remove the leaf from the syringe. Gently but thoroughly blot the leaf with absorbent paper to remove surface liquid. 
9. Measure the weight of the infiltrated leaf. Calculate the difference in weight before and after infiltration which closely approximates the infiltration volume.

3. Leaf infiltration by vacuum flask:

1. Detach the leaf from the plant at the petiole using a razor blade.

2. Rinse the freshly excised leaf by submersion in distilled water to remove leaf surface contaminants. Dry the leaf by gently blotting with absorbent paper.

3. Measure the leaf weight before infiltration.

4. Place the leaf (or leaves if infiltrating multiple leaves) in a $250 \mathrm{ml}$ or larger side arm flask containing distilled water (other infiltration fluids may be used, see Discussion). Fully cover the leaves with the liquid.

5. Apply a vacuum to the flask. Gently agitate to release the air bubbles from the leaves. Carefully and slowly release the vacuum. NOTE: Quickly releasing the vacuum may result in cell lysis and cytoplasmic contamination.

6. Repeat step 1.3.5 until the leaf is fully infiltrated, as seen by the darkened colour of the infiltrated areas.

7. Remove the leaf from the flask. Gently but thoroughly blot the leaf with absorbent paper to remove surface liquid.

8. Measure the weight of the infiltrated leaf. Calculate the difference in weight before and after infiltration which closely approximates the infiltration volume.

4. Recovery of AWF by centrifugation:

1. Place the leaf on a piece of 4 inch $(10 \mathrm{~cm}$ ) wide Parafilm. Using a $5 \mathrm{ml}$ pipette tip (or a similar sized object), roll up the leaf within the Parafilm.

2. Insert the rolled up leaf with pipette tip into a $20 \mathrm{ml}$ syringe. Position any cut leaf edges facing upwards. Insert the syringe into a clean $50 \mathrm{ml}$ polyethylene or polycarbonate tube.

3. Centrifuge the syringe within the tube for $10 \mathrm{~min}$ at $1,000 \mathrm{xg}$ in a swinging bucket rotor at $4{ }^{\circ} \mathrm{C}$. NOTE: Visual examination of the leaves following centrifugation should reveal that the majority of the infiltration fluid has been expelled. Darker green patches indicate that additional centrifugation time is required.

4. Pipette the recovered AWF into fresh $1.5 \mathrm{ml}$ tubes.

NOTE: The volume of AWF should approximately match the infiltration volume for that leaf; if the volume is less then additional centrifugation time or centrifugation speed is required.

5. Centrifuge the AWF samples at $15,000 \times \mathrm{g}$ for $5 \mathrm{~min}$ to remove any cells or particulate matter. Alternatively, pass the AWF through a $0.22 \mu \mathrm{m}$ filter to remove cells and particulate matter.

6. Pipette the supernatant into a fresh tube. Keep samples on ice until storage.

7. Store the AWF samples at $-80^{\circ} \mathrm{C}$.

\section{Assays for Cytoplasmic Contamination}

1. Keep the AWF samples on ice to minimize enzymatic reactions (e.g., proteolysis) and perform the assays immediately after the centrifugation steps are complete.

NOTE: For metabolite assays, samples may be immediately frozen after extraction and stored at $-80^{\circ} \mathrm{C}$ until use.

2. For a standard malate dehydrogenase $(\mathrm{MDH})$ assay protocol see ${ }^{15}$; otherwise use a commercially available $\mathrm{MDH}$ assay kit.

3. For a standard glucose-6-phosphate dehydrogenase (G6PDH) assay protocol see ${ }^{16}$; otherwise use a commercially available G6PDH assay kit.

4. For quantification of cytoplasmic metabolites such as glucose-6-phosphate (G-6-P) use GC-MS as described in step 5. Otherwise, use a commercially available kit for the direct assay of G-6-P.

\section{Calculation of the Apoplast Dilution Factor}

1. Prepare two volumes of the infiltration fluid used above in step 1.2.4 or 1.3.4 (e.g., distilled water). Add indigo carmine powder to one solution to a final concentration of $50 \mu \mathrm{M}$, add nothing to the other.

2. Measure the absorbance at $610 \mathrm{~nm}\left(\mathrm{OD}_{610}\right)$ of $200 \mu \mathrm{l}$ of the indigo carmine infiltration solution with a plate reader. Correct this value by subtracting the $\mathrm{OD}_{610}$ of $200 \mu \mathrm{l}$ of infiltration solution without indigo carmine. Substitute this corrected value as $\mathrm{OD}_{610 \text { infiltrate }}$ into the equation below.

3. Perform at least three replicate leaf infiltrations as above (step $\mathbf{1 . 2}$ or 1.3) with both infiltration solutions (with and without indigo carmine).

4. After infiltration, rinse the leaves in distilled water to remove any remaining indigo carmine present on the outer surfaces.

5. Proceed with centrifugation as above (step 1.4).

6. Determine the average $O_{610}$ of $200 \mu \mathrm{l}$ of the indigo carmine AWF extractions. Correct this value by subtracting the average $\mathrm{OD}_{610}$ value of $200 \mu \mathrm{l}$ of indigo carmine free AWF. Substitute this corrected value as $\mathrm{OD}_{610 \mathrm{AWF}}$ into the equation below.

7. Calculate the dilution factor (i.e., fold dilution) from the corrected absorbance values as: Apoplast dilution factor $=\mathrm{OD}_{610 \text { infiltrate }} /\left(\mathrm{OD}_{610 \text { infiltrate }}-\mathrm{OD}_{610 \mathrm{AWF}}\right)$

8. Multiply concentration or activity values from AWF by the dilution factor to estimate the concentration/activity in apoplastic fluid in planta.

\section{Concentration of AWF to Full Strength by Freeze Drying}

1. Turn on the freeze-drier and allow it to stabilize at the working temperature and pressure.

2. Pool the desired amount of fresh or stored AWF samples.

3. Distribute the samples evenly among $2 \mathrm{ml}$ centrifuge tubes.

4. Determine the reconstitution volume:

Reconstitution volume $=$ volume before freeze-drying $/$ apoplast dilution factor 
5. With the lids closed, freeze the tubes in liquid nitrogen.

6. Transfer the frozen tubes to one or more chilled freeze-drying vessels. While transferring the tubes, replace the lid with one that has been pierced with a sharp object to allow gas exchange.

7. Attach the vessels to the freeze-drier and lyophilize the samples completely.

8. Remove the samples from the machine and reconstitute the AWF by adding the calculated amount of distilled water.

9. Replace the unpierced lid and store the samples at $-80^{\circ} \mathrm{C}$.

\section{Metabolite Analysis by Gas Chromatography Mass Spectrometry ${ }^{17}$}

1. Pipette $200 \mu \mathrm{l}$ of AWF into a $1.5 \mathrm{ml}$ tube

2. Add $700 \mu \mathrm{l}$ of methanol containing $10 \mu \mathrm{g} / \mathrm{ml}$ ribitol as an internal standard.

3. Heat at $70^{\circ} \mathrm{C}$ for $10 \mathrm{~min}$.

4. Centrifuge for $10 \mathrm{~min}$ at $11,000 \times \mathrm{g}$.

5. Transfer $700 \mu \mathrm{l}$ of supernatant to a fresh $1.5 \mathrm{ml}$ tube.

6. Add $375 \mu \mathrm{l}$ of cold chloroform and vortex for $10 \mathrm{sec}$.

7. Add $500 \mu \mathrm{l}$ of $\mathrm{dH}_{2} \mathrm{O}$ and vortex for $10 \mathrm{sec}$.

8. Centrifuge for $15 \mathrm{~min}$ at $2,200 \mathrm{xg}$.

9. Transfer $150 \mu \mathrm{l}$ of the upper aqueous layer into a new $1.5 \mathrm{ml}$ tube, then dry the samples completely in a vacuum concentrator without heat.

10. Dissolve the samples in $30 \mu \mathrm{l}$ of pyridine containing $20 \mathrm{mg} / \mathrm{ml}$ methoxyamine hydrochloride.

11. Incubate at $70^{\circ} \mathrm{C}$ while shaking vigorously for $2 \mathrm{hr}$

12. Add $50 \mu$ l of MSTFA (N-methyl-N-(trimethylsilyl) trifluoroacetamide).

13. Incubate at $37^{\circ} \mathrm{C}$ while shaking for $30 \mathrm{~min}$.

14. Transfer samples to GC-MS compatible glass vials.

15. Perform GC-MS analysis of the samples. Refer to Lisec et al. $2009^{17}$, for details on GC-MS equipment setup and performance.

\section{Representative Results}

Typical results for AWF extractions performed on healthy 3 week old $P$. vulgaris cv. Tendergreen/eaves, using distilled water as the infiltration fluid are shown in Table 2. Young $P$. vulgaris leaves are amenable to infiltration, and at the centrifugation speed used here $(1,000 \times \mathrm{g})$ the removal of the majority of infiltration fluid by centrifugation can be seen directly as the leaves revert to their original green colour. $P$. vulgaris leaf fresh weight was on average $1.1 \mathrm{x}$ g before infiltration and yielded $0.5 \mathrm{ml}$ of AWF per gram fresh weight. The protein concentration of the AWF was determined to be $0.18 \pm 0.08 \mathrm{mg} / \mathrm{ml}$ using a standard Bradford protein assay. The dilution factor for these leaves, calculated by measuring indigo carmine dilution, was $2.3 \pm 0.3$ fold. The above values can vary substantially between species and pilot experiments are required to determine the yield of AWF per gram leaf fresh weight, as well as the AWF protein concentration and dilution factor that can be expected for a given leaf type.

Damage to the integrity of the cells can occur both during the infiltration step, if the changes in pressure are too rapid, and during the centrifugation step if the centrifugal force is too high. It is therefore necessary to assay AWF samples for markers of cytoplasmic contamination; ideally, multiple independent assays are performed. Here, the results from three possible assays are reported in Table 2. In well-performed extractions of $P$. vulgaris AWF, G6PDH was undetectable by a standard enzyme assay. This is consistent with other reports where G6PDH activity becomes detectable only above a threshold centrifugal force ${ }^{12}$. In contrast, a baseline level of malate dehydrogenase activity $(2.5 \mathrm{U} /$ $\mathrm{ml}$ ) was detectable in all $P$. vulgaris AWF samples using a standard metabolic assay. Increasing the centrifugal force above a threshold of $1,000 \times \mathrm{g}$ resulted in increased MDH activities (results not shown). As the apoplast from other species is known to contain endogenous MDH activity ${ }^{18}$, the amount detected here is considered genuine apoplastic activity and significant increases above this baseline level are indicative of contamination. Metabolites that are thought to be predominantly cytoplasmic, such as hexose-6-phosphates, can also be used to assess contamination of AWF. Here, GC-MS analysis detected zero or trace levels of G-6-P in AWF extractions. By contrast, in cut maize root sections G-6-P was detected after AWF extraction at all centrifugation speeds and increased in concentration at higher speeds, indicating cytoplasmic contamination $^{10}$.

Metabolite analysis by GC-MS of $P$. vulgaris leaf AWF typically yields 40 - 60 identifiable metabolites and an approximately equal number of unidentifiable compounds (Figure 2). Organic acids, simple sugars, and amino acids represent the bulk of the identifiable metabolites, however, secondary plant metabolites have also been detected and quantified from AWF ${ }^{11}$. Several example peaks from these different molecule classes are labeled in Figure 2. Further downstream analytical techniques are applicable to these AWF samples to quantify various molecules, for example: ICP-MS, NMR, HPLC, atomic absorption spectroscopy, protein mass spectrometry.

The protein component of the apoplast is also represented in the AWF as shown in the Coomassie Brilliant Blue stained SDS-PAGE gel in Figure 3. Among other roles, the apoplastic enzymes are responsible for the synthesis of the cell wall and the creation of extracellular reactive oxygen species. Proteomic studies of AWF from various species have identified dozens of individual proteins and shown that the protein component of the apoplast responds to environmental stress ${ }^{13,19}$. Ideally AWF extract should be free from contamination by cytoplasmic proteins such as Rubisco; however in practice this is difficult to achieve. The presence of a Rubisco protein band at $\sim 53 \mathrm{kDa}$ following SDS-PAGE provides a further qualitative assay for the integrity of AWF samples. For example, the sample loaded in lane 2 in Figure 3 contains a greater amount of Rubisco contamination that of lane 1. 


\begin{tabular}{|l|l|}
\hline & Phaseolus vulgaris AWF \\
\hline Volume of AWF $\left(\mathrm{ml} \mathrm{g}^{-1}\right.$ leaf $\left.\mathrm{FW}\right)$ & $0.49 \pm 0.09$ \\
\hline Protein conc. $\left(\mathrm{mg} \mathrm{ml}^{-1}\right)$ & $0.18 \pm 0.08$ \\
\hline Dilution factor & $2.3 \pm 0.3$ \\
\hline G6PDH activity $\left(\mathrm{U} \mathrm{ml}^{-1}\right)$ & none detected \\
\hline Glc-6-P $\left(\mathrm{mg} \mathrm{ml}^{-1}\right)$ & none detected \\
\hline MDH activity $\left(\mathrm{U} \mathrm{ml}^{-1}\right)$ & $2.5 \pm 0.9$ \\
\hline
\end{tabular}

Table 2. Typical results for AWF extractions from $P$. vulgaris cv. Tendergreen leaves.

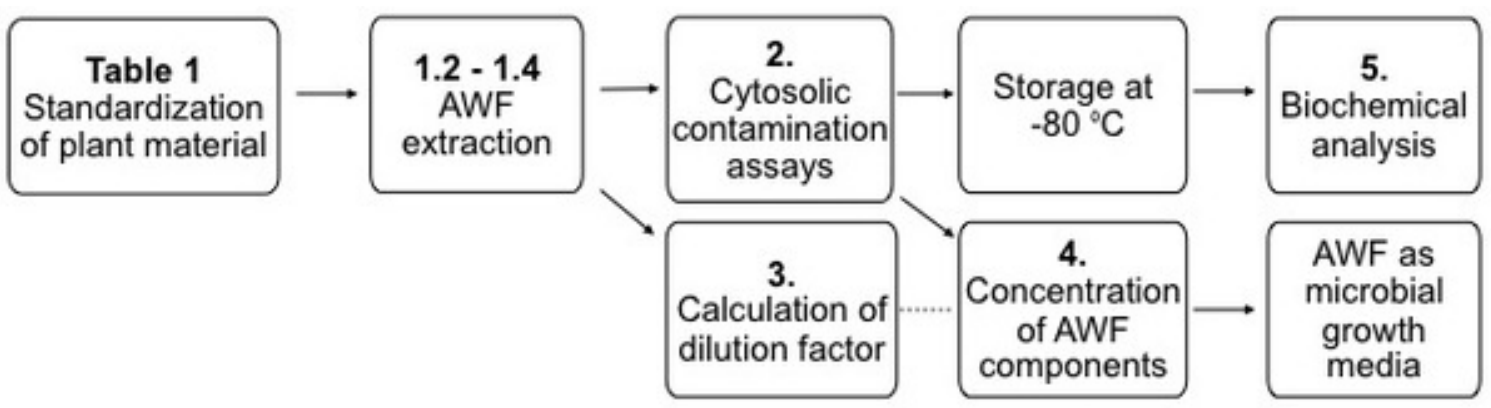

Figure 1. Standard apoplast extraction workflow. The numbers refer to steps in the protocol.

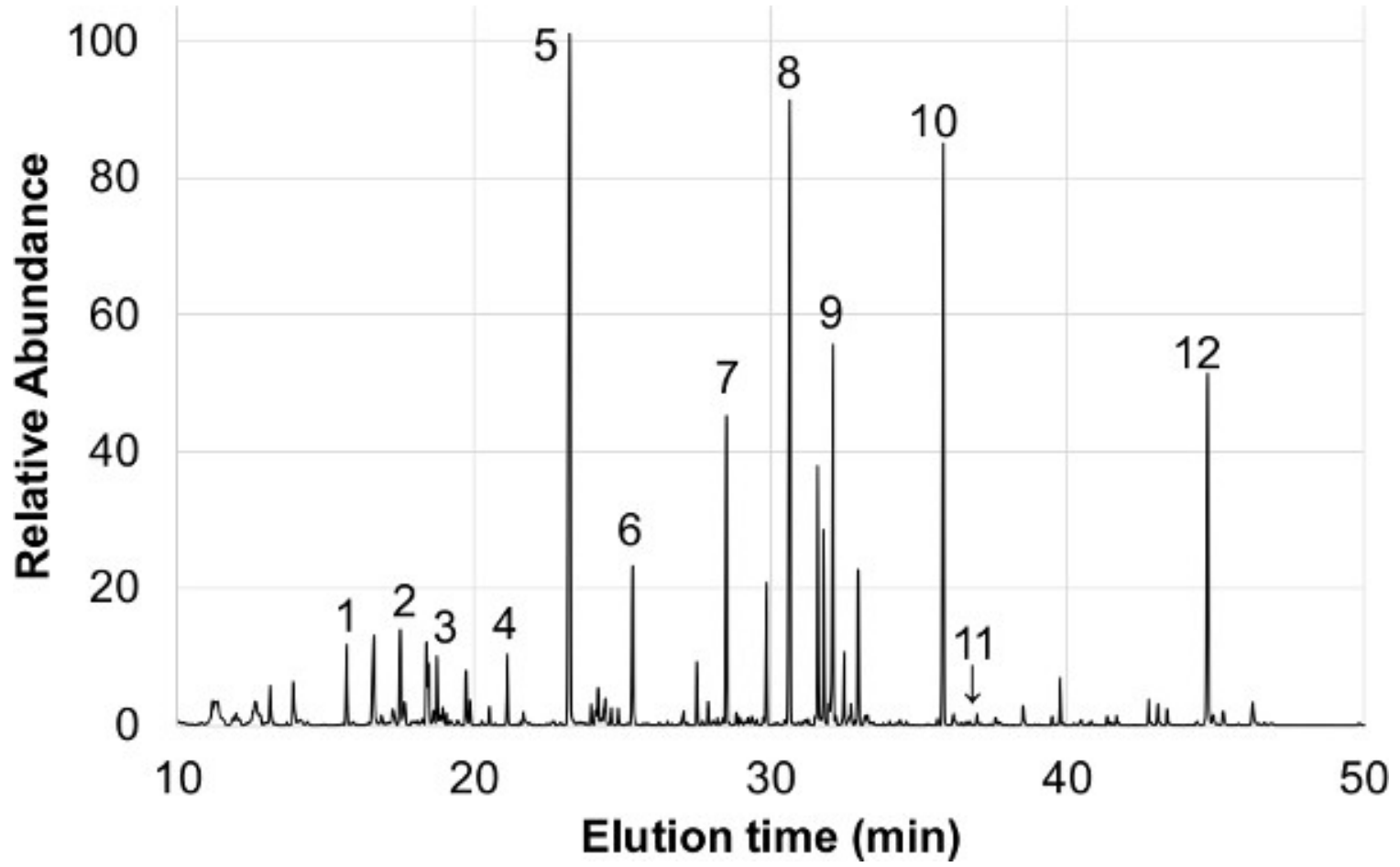

Figure 2. An example GC-MS chromatogram of $\boldsymbol{P}$. vulgaris cv. Tendergreen AWF. The numbered example metabolite peaks are: 1 malonate, 2-phosphate, 3-succinate, 4-unknown, 5-malate, 6-asparagine, 7-ribitol (internal standard), 8-citrate, 9-glucose, 10-inositol, 11-caffeic acid, 12-sucrose. 


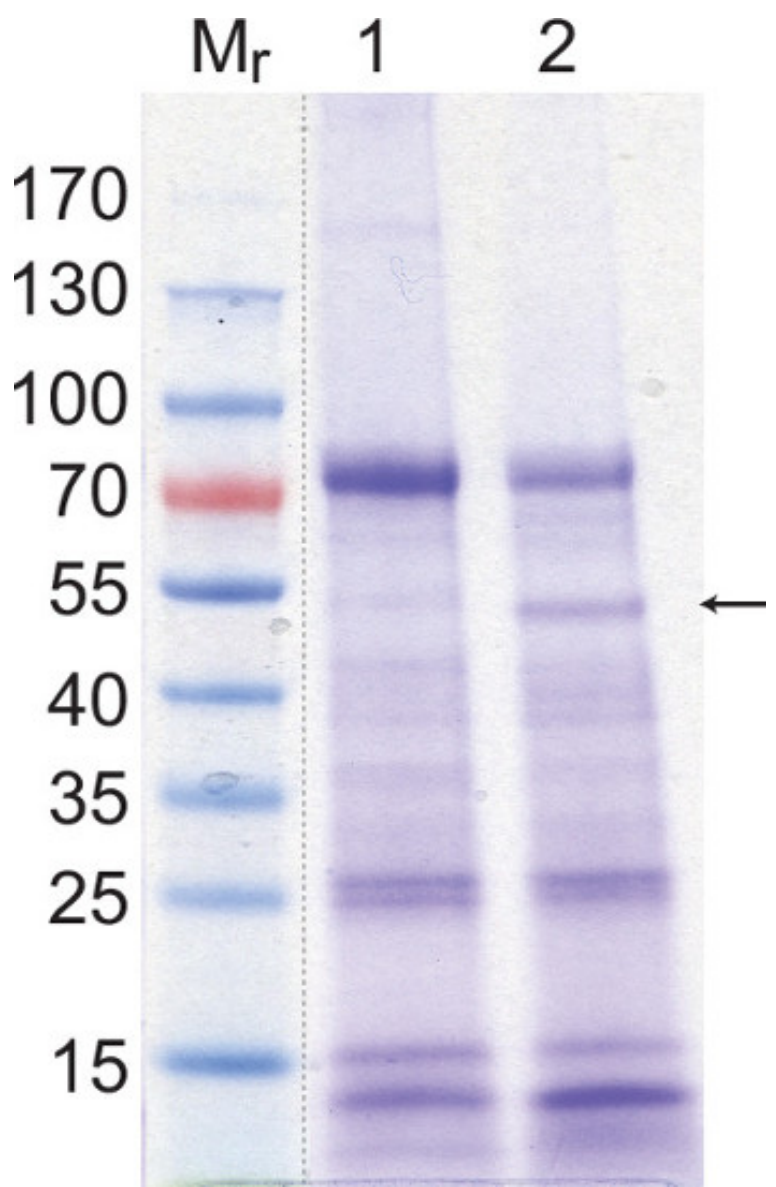

Figure 3. An example SDS-PAGE Coomassie stained gel of $\boldsymbol{P}$. vulgaris AWF leaf extracts. This gel provides a comparison between the protein components of two AWF extractions that differ in the amount of cytoplasmic contamination by the abundant plastidial enzyme Rubisco. Following AWF extraction both samples were subjected to acetone protein precipitation in a 4 fold excess ( $/ \mathrm{v}$ ) of cold acetone and resolubilized in water at one tenth the original volume. Lanes 1 and 2 contain $40 \mu \mathrm{g}$ of protein. The band corresponding to the Rubisco large chain ( $53 \mathrm{kDa})$ is indicated by an arrow. $\mathrm{M}_{\mathrm{r}}$ : protein molecular weight markers.

\section{Discussion}

\section{Optimizing the plant tissue source}

Biological and technical variation can be large when performing apoplast extractions, thus a highly standardized workflow is helpful to increase continuity across experiments (Figure 1). Importantly, the source of plant tissue must be standardized, including leaf type, leaf age, growth/ environmental conditions and time of day (Table 1). Large differences exist in the ease with which different leaves are infiltrated and the AWF subsequently recovered by centrifugation; these differences are correlated with stomata number, aperture size and mesophyll resistance ${ }^{12,14}$. Even among different cultivars of $P$. vulgaris there are large differences in the ease and yield of the AWF extraction procedure; for example leaves of the Tendergreen variety used here are more amenable to AWF extraction using this method than the Canadian Wonder cultivar. Within $P$. vulgaris the first true leaves are the biggest and the easiest to infiltrate, making them the obvious choice for apoplastic extractions. The apoplastic air and water volume has been shown to vary with leaf age in several species leading to differences in AWF extractability ${ }^{12,14}$. In $P$. vulgaris, older leaves become substantially more difficult to infiltrate and yield less AWF upon centrifugation; therefore leaves were harvested when they reached full expansion. Leaves which are difficult to infiltrate subsequently require higher centrifugation speeds to recover the AWF. One should therefore carefully screen several different leaf types and varieties before deciding upon a tissue source for large scale AWF extractions.

Plant growth conditions must also be standardized as much as possible within the context of the experiment. The use of growth cabinets is preferable as they allow consistent humidity, temperature and light intensity regiments to be maintained. The harvesting of leaves should always occur at the same time of day because the concentrations of metabolites, enzymes, etc. vary throughout the diurnal cycle ${ }^{14}$. Finally, to ensure that the plant leaves all have similar turgor pressure, the plants should be watered soon before $(\sim 1 \mathrm{hr})$ the harvest.

\section{Optimization of leaf infiltration and centrifugation}

Undesirable cytoplasmic contamination of the AWF due to partial cell lysis will result if the mechanical stress encountered by the cells is too high during the infiltration or centrifugation steps. Therefore, the procedure for a given leaf type should be an optimized trade-off between maximizing yield and minimizing cytoplasmic contamination of the AWF. In all cases, one should use the lowest centrifugation speed at which AWF can be recovered to avoid possible mechanical disruption of the leaf cells. Optimization of the centrifugation speed should be determined 
empirically for each leaf type by monitoring the volume recovered and apoplast contamination over a range of centrifugation speeds. It has been observed that marker enzyme activities, such as MDH, G6PDH and glucose-phosphate isomerase, remain low until a threshold centrifugal force is surpassed, above which these activities increase rapidly, presumably due to cytoplasmic leakage ${ }^{9,12,14}$. The use of Parafilm for support during the centrifugation step, as noted by Baker et al. $2012^{11}$, can improve the efficacy of the AWF extraction and may minimize mechanical damage to the leaf blade caused by excessive folding and compression. Furthermore, the use of Parafilm improves visualization of the leaf after centrifugation when one should examine the leaf for damage and completeness of the AWF extraction.

Nouchi ${ }^{12}$ describe the optimization of AWF recovery from cut rice leaf sections, which are difficult to infiltrate and require higher centrifugation speeds to collect AWF because of their small stomatal openings. Improved wetting of the rice leaf surface, either by presoaking the leaves in distilled water or the addition of a surfactant to the infiltration fluid, facilitated the infiltration process. A higher centrifugation speed $(6,000$ $\mathrm{xg}$ ) was also used while monitoring for apoplastic contamination ${ }^{12}$. When using cut leaf sections there is always the risk that cytoplasmic contamination will be more prevalent even with extensive washing of the wound sites; cut leaves should therefore only be used when necessary.

For many studies distilled water is used as the infiltration fluid ${ }^{11}$. However, compounds may be added to the infiltration fluid, such as salts or buffers, to improve the extraction of certain apoplastic compounds, especially proteins ${ }^{13}$. Lohaus ${ }^{14}$ evaluated the effect of ionic and osmotic strength on the composition of the recovered AWF and found them to be negligible. Changes in $\mathrm{pH}$ of the infiltration medium, however, may affect the AWF composition ${ }^{14}$.

Proper handling and storage of the extracted apoplastic fluid is important. AWF has been shown to contain an abundance of proteases and other enzymes $^{13,20}$, as well as volatile organic compounds. Therefore, to reduce changes to the composition of AWF after recovery it is recommended to keep samples on ice or otherwise stored at $-80^{\circ} \mathrm{C}$. Furthermore, enzymatic assays of AWF should be performed as soon as possible after extraction to limit enzyme inactivation due to proteolysis, prolonged dilution or freeze-thawing.

The process of infiltration dilutes the apoplastic fluid and it is often necessary to determine the extent of this dilution. The centrifugation step may also dilute the AWF with water from intracellular compartments. A dilution factor is needed to estimate in vivo apoplast chemical concentrations when measurements are made on AWF. A dilution factor is also needed to concentrate AWF back to full strength when it is being used as an apoplast mimicking growth medium for microbes - thus matching in vivo metabolite concentrations as accurately as possible. Several variations exist on the method described in step $\mathbf{4}$ for determining the AWF dilution factor by measuring the dilution of a marker compound added to the infiltration fluid. For all methods the AWF dilution calculation assumes that the infiltration fluid is not appreciably absorbed or diluted by the leaf cells during the AWF recovery process. This assumption has been previously verified for the infiltration step ${ }^{14}$ but is unverified for the centrifugation step. The marker compound must also not be absorbed, transported or modified while in the apoplast. Indigo carmine is the most widely used and thoroughly tested of dyes used for AWF dilution calculations. Indigo carmine displays a low absorbance to cation exchange resin and isolated cell walls and was shown to be suitable for AWF calculations in Brassica napus, Pisum sativum, Solanum lycopersicum and Glycine $\max ^{11,21,22}$. However, in some leaf types, e.g., rice and cucumber, indigo carmine appeared not be fully recovered after infiltration, which would lead to an underestimation of the AWF dilution factor ${ }^{12,21}$. The lack of recovery of indigo carmine may be due to its susceptibility to cleavage into isatin sulfonate by superoxide ${ }^{23}$, which is known to be produced in the apoplast, especially under stress ${ }^{24}$. Cleavage of indigo carmine into isatin sulfonate will result in a loss of absorbance at $610 \mathrm{~nm}$ and an increase at $245 \mathrm{~nm}$. Whether this reaction occurs to an appreciable extent in the apoplast, or whether this reaction can be inhibited by the addition of superoxide scavengers to infiltration fluid has not been investigated to date. Blue dextran has been used instead of indigo carmine for quantification of the AWF dilution factor in rice leaves ${ }^{12}$ though its stability and recovery in AWF has not been reported. Alternatively, radiolabelled compounds such as $\left[{ }^{14} \mathrm{C}\right]$ sorbitol or other quantifiable internal standards can be used instead of dyes and the decrease in radioactivity or concentration measured and used to calculate the dilution factor in an analogous way ${ }^{11,14,25}$.

\section{Limitations of the technique}

A few caveats exist for the infiltration-centrifugation AWF isolation method. First, the dilution of the apoplast during infiltration may elicit a response from the plant. If the surrounding leaf cells detect a diminished concentration for certain components of the apoplastic fluid, they may respond by excreting further metabolites, distorting the interpretation of metabolite concentrations. In an assessment of this problem it was observed that neither the time between infiltration and centrifugation nor moderate differences in the ionic strength of the infiltration fluid affected the composition of the extracted AWF ${ }^{14,22}$. Therefore, any artifacts resulting from apoplast dilution are thought to be minimal.

A second potential drawback is that elution of AWF by centrifugation does not capture all the molecules present in the apoplast for several reasons. Some compounds, in particular cations and proteins may be tightly associated with the negatively charged cell wall and not elute with the $\mathrm{AWF}^{13}$. Other molecules such as proteins may be too large to elute efficiently out of the apoplast at the centrifugation speeds used ${ }^{14}$. Reactive oxygen species are an important class of compound produced in the apoplast, but due to the short-lived nature of these compounds, and unspecified requirements for their production, their presence is not well captured by AWF extraction. It is not known how accurately AWF represents the in vivo composition of the apoplast fluid and this may vary between species.

Several assays exist to determine cytoplasmic contamination, though none are universally accepted. Assays of predominantly cytoplasmic enzymes (e.g., G6PDH, hexose phosphate isomerase, $\mathrm{MDH}$ ) have the benefit of being easy to perform but may not correlate well with cytoplasmic leakage ${ }^{14,18}$. The assessment of cytoplasmic metabolites (e.g., hexose phosphates, chlorophyll) is perhaps more indicative but is less well established ${ }^{11}$. Nevertheless, either type of assay can be useful for relative quantification of apoplastic contamination between samples. Ideally, multiple independent measurements should be used to validate the integrity of the AWF sample.

While acknowledging its limitations, the infiltration-centrifugation technique described here remains a simple and robust technique in the study of apoplastic proteins, primary and secondary metabolites and inorganic ions. 


\section{Disclosures}

No conflicts of interest declared.

\section{Acknowledgements}

This work was supported by grants BB/J016012/1 and BB/E007872/1 from the UK Biotechnology and Biological Sciences Research Council (BBSRC) to Gail Preston.

\section{References}

1. Felle, H. H. Apoplastic pH during low-oxygen stress in barley. Annals of Botany. 98 (5), 1085-93, doi: 10.1093/aob/mcl193 (2006).

2. Schaarschmidt, S., Kopka, J., Ludwig-Müller, J., \& Hause, B. Regulation of arbuscular mycorrhization by apoplastic invertases: enhanced invertase activity in the leaf apoplast affects the symbiotic interaction. The Plant Journal. 51 (3), 390-405, doi: 10.1111/ j.1365-313X.2007.03150.x (2007)

3. Outlaw, W. H., \& De Vlieghere-He, X. Transpiration rate. An important factor controlling the sucrose content of the guard cell apoplast of broad bean. Plant Physiology. 126 (4), 1716-24 (2001).

4. Rico, A., Jones, R., \& Preston, G. M. Adaptation to the plant apoplast by plant pathogenic bacteria. Plant Pathogenic Bacteria. 63-89 (2009).

5. Rico, A., \& Preston, G. M. Pseudomonas syringae pv. tomato DC3000 uses constitutive and apoplast-induced nutrient assimilation pathways to catabolize nutrients that are abundant in the tomato apoplast. Molecular Plant-Microbe Interactions. 21 (2), 269-82, doi: 10.1094/ MPMI-21-2-0269 (2008).

6. Solomon, P., Tan, K., \& Oliver, R. The nutrient supply of pathogenic fungi; a fertile field for study. Molecular Plant Pathology. 4, 203-210, doi: 10.1046/j.1364-3703.2003.00161.x. (2003).

7. Brunner, F. Innate immunity in plants and animals: emerging parallels between the recognition of general elicitors and pathogen-associated molecular patterns. Current Opinion in Plant Biology. 5 (4), 318-324, doi: 10.1016/S1369-5266(02)00265-0 (2002).

8. Fontaniella, B., Vicente, C., Armas, R., \& Legaz, M. E. Effect of leaf scald (Xanthomonas albilineans) on polyamine and phenolic acid metabolism of two sugarcane cultivars. European Journal of Plant Pathology. 119 (4), 401-409, doi: 10.1007/s10658-007-9172-2 (2007).

9. Millán, A. F., Morales, F., Abadía, A., \& Abadía, J. Effects of iron deficiency on the composition of the leaf apoplastic fluid and xylem sap in sugar beet. Implications for iron and carbon transport. Plant Physiology. 124 (2), 873-84 (2000).

10. Dragišić Maksimović, J. L., et al. Filter strip as a method of choice for apoplastic fluid extraction from maize roots. Plant Science. 223 (1), 49-58 (2014)

11. Baker, C. J., et al. An internal standard technique for improved quantitative analysis of apoplastic metabolites in tomato leaves. Physiological and Molecular Plant Pathology. 78, 31-37, doi: 10.1016/j.pmpp.2012.01.001 (2012).

12. Nouchi, I., et al. Overcoming the difficulties in collecting apoplastic fluid from rice leaves by the infiltration-centrifugation method. Plant \& Cell Physiology. 53 (9), 1659-68, doi: 10.1093/pcp/pcs102 (2012).

13. Boudart, G., et al. Cell wall proteins in apoplastic fluids of Arabidopsis thaliana rosettes: identification by mass spectrometry and bioinformatics. Proteomics. 5 (1), 212-21, doi: 10.1002/pmic.200400882 (2005).

14. Lohaus, G., Pennewiss, K., Sattelmacher, B., Hussmann, M., \& Hermann Muehling, K. Is the infiltration-centrifugation technique appropriate for the isolation of apoplastic fluid? A critical evaluation with different plant species. Physiologia Plantarum. 111 (4), $457-465$ (2001).

15. Bergmeyer, H. U. Malate Dehydrogenase. Methods of Enzymatic Analysis Volume 2. 614 (1974).

16. Lohr, G. W., \& Waller, H. D. Glucose-6-phosphate Dehydrogenase. Methods of Enzymatic Analysis Volume 2. 636-643 (1974).

17. Lisec, J., Schauer, N., Kopka, J., Willmitzer, L., \& Fernie, A. R. Gas chromatography mass spectrometry-based metabolite profiling in plants. Nature Protocols. 1 (1), 387-96, doi: 10.1038/nprot.2006.59 (2006).

18. Li, Z. C., McClure, J. W., \& Hagerman, A. E. Soluble and bound apoplastic activity for peroxidase, beta-d-glucosidase, malate dehydrogenase, and nonspecific arylesterase, in barley (Hordeum vulgare L.) and oat (Avena sativa L.) primary leaves. Plant Physiology. 90 (1), 185-90 (1989).

19. Dani, V., Simon, W. J., Duranti, M., \& Croy, R. R. D. Changes in the tobacco leaf apoplast proteome in response to salt stress. Proteomics. $\mathbf{5}$ (3), 737-45, doi: 10.1002/pmic.200401119 (2005).

20. Shabab, M. et al. Fungal effector protein AVR2 targets diversifying defense-related Cys proteases of tomato. Plant Cell. 20 (4), 1169-83 (2008).

21. Cosgrove, D. J., \& Cleland, R. E. Solutes in the free space of growing stem tissues. Plant Physiology. 72 (2), $326-31$ (1983).

22. Husted, S., \& Schjoerring, J. K. Apoplastic pH and ammonium concentration in leaves of Brassica napus L. Plant Physiology. 109 (4), 145360 (1995).

23. Kettle, A. J., Clark B. M., \& Winterbourn C. C. Superoxide converts indigo carmine to isatin sulfonic acid. The Journal of Biological Chemistry. 279 (18), 18521-5 (2004).

24. Bournonville, C. F. G., \& Díaz-Ricci, J. C. Quantitative determination of superoxide in plant leaves using a modified NBT staining method. Phytochemical Analysis. 22 (3), 268-71 (2011).

25. Speer, M., \& Kaiser, W. M. Ion relations of symplastic and apoplastic space in leaves from Spinacia oleracea L. and Pisum sativum L. under salinity. Plant Physiology. 97 (3), 990-7 (1991). 